PROCEEDINGS OF THE

AMERICAN MATHEMATICAL SOCIETY

Volume 132, Number 3, Pages 875-883

S 0002-9939(03)07097-7

Article electronically published on August 28, 2003

\title{
INTEGRATION BY PARTS ON THE BROWNIAN MEANDER
}

\author{
STEFANO BONACCORSI AND LORENZO ZAMBOTTI
}

(Communicated by Claudia M. Neuhauser)

\begin{abstract}
We prove infinite-dimensional integration by parts formulae for the laws of the Brownian Meander, of the Bessel Bridge of dimension 3 between $z, z^{\prime} \geq 0$ and of the Brownian Motion on the set of all paths taking values greater than or equal to a nonpositive constant. We give applications to SPDEs with reflection.
\end{abstract}

\section{INTRODUCTION AND MAIN RESULT}

Let $(B(t))_{t \in[0,1]},(M(t))_{t \in[0,1]}$ and $\left(b_{z}^{z^{\prime}}(t)\right)_{t \in[0,1]}$ be, respectively, a Brownian motion, a Brownian Meander and a Bessel Bridge of dimension 3 between $z$ and $z^{\prime} \geq 0$ over the time interval $[0,1]$; see [6]. All the processes here are defined on a complete probability space $(\Omega, \mathcal{F}, \mathbb{P})$.

The aim of this paper is to prove the infinite-dimensional integration by parts formulae (1.2) - (1.4) below for the laws of $(M(t))_{t \in[0,1]},\left(b_{z}^{z^{\prime}}(t)\right)_{t \in[0,1]}$ and $(B(t))_{t \in[0,1]}$ on the convex set of paths $K_{\alpha}:=\{x:[0,1] \mapsto[-\alpha, \infty)$ continuous $\}, \alpha \geq 0$. In the last section, we also give applications of (1.2)-(1.4) to SPDEs with reflection. In the spirit of [7], we identify the reflecting terms in the SPDEs as families of additive functionals whose Revuz measures are equal to the boundary measures of (1.2) (1.4) .

We introduce the space $E:=\{u \in C([0,1]): u(0)=0\}$ and define the operator $V:[0,1] \times E \times E \mapsto E:$

$$
\begin{aligned}
V(r, f, g)(t):=-\sqrt{r} f(1)+\sqrt{r} & 1_{[0, r]}(t) f\left(1-\frac{t}{r}\right) \\
& +\sqrt{1-r} 1_{[r, 1]}(t) g\left(\frac{t-r}{1-r}\right), \quad t \in[0,1] .
\end{aligned}
$$

Then, the main result of the paper is the following:

Theorem 1.1. Let $H:=L^{2}(0,1)$, endowed with the canonical scalar product $\langle\cdot, \cdot\rangle$. For all maps $\varphi: H \mapsto \mathbb{R}$, bounded with bounded continuous Fréchet differential, and for all $h \in C^{2}([0,1])$, we denote by $h^{\prime \prime} \in H$ the second derivative of $h$ and by $\partial_{h} \varphi$, the directional derivative of $\varphi$ along $h$.

Received by the editors June 1, 2002 and, in revised form, October 28, 2002.

2000 Mathematics Subject Classification. Primary 60H07, 60H15, 60J55; Secondary 31C25.

Key words and phrases. Integration by parts, Brownian motion, stochastic partial differential equations with reflection. 
Let $\left(b_{0}^{0}, M\right)$ be an independent couple of processes. Then for all $h \in C_{c}^{2}((0,1])$ :

$$
\begin{aligned}
& \mathbb{E}\left[\partial_{h} \varphi(M)\right]=-\mathbb{E}\left[\varphi(M)\left\langle M, h^{\prime \prime}\right\rangle\right] \\
& \quad-\int_{0}^{1} d r h(r) \frac{1}{\sqrt{2 \pi r^{3}(1-r)}} \mathbb{E}\left[\varphi\left(V\left(r, b_{0}^{0}, M\right)\right)\right] .
\end{aligned}
$$

Now let $\left(b_{0}^{z}, M\right)$ be an independent couple for all $z \geq 0$. Then for all $h \in C_{c}^{2}((0,1])$ :

$$
\begin{aligned}
\mathbb{E}\left[\partial_{h} \varphi(B) 1_{K_{\alpha}}(B)\right]= & -\mathbb{E}\left[\varphi(B)\left\langle B, h^{\prime \prime}\right\rangle 1_{K_{\alpha}}(B)\right] \\
& -\int_{0}^{1} d r h(r) \frac{\alpha \exp \left(-\frac{\alpha^{2}}{2 r}\right)}{\pi \sqrt{r^{3}(1-r)}} \mathbb{E}\left[\varphi\left(V\left(r, b_{0}^{\alpha / \sqrt{r}}, M\right)\right)\right] .
\end{aligned}
$$

Finally, let $\left(b_{0}^{z}, \hat{b}_{0}^{z^{\prime}}\right)$ be an independent couple for all $z, z^{\prime} \geq 0$, and assume by convention that $0 /(1-\exp (0))=1$. Then for all $k \in C_{c}^{2}(0,1)$,

$$
\begin{gathered}
\mathbb{E}\left[\partial_{k} \varphi\left(b_{z}^{z^{\prime}}\right)\right]=-\mathbb{E}\left[\varphi\left(b_{z}^{z^{\prime}}\right)\left\langle b_{z}^{z^{\prime}}, k^{\prime \prime}\right\rangle\right] \\
-\int_{0}^{1} k(r) \frac{\exp \left(-\frac{z^{2}}{2} \frac{r}{1-r}-\frac{\left(z^{\prime}\right)^{2}}{2} \frac{1-r}{r}-2 z z^{\prime}\right)}{\sqrt{2 \pi r^{3}(1-r)^{3}}} \frac{2 z z^{\prime}}{1-\exp \left(-2 z z^{\prime}\right)} . \\
\cdot \mathbb{E}\left[\varphi\left(z+V\left(r, b_{0}^{z / \sqrt{r}}, \hat{b}_{0}^{z^{\prime} / \sqrt{1-r}}\right)\right)\right] d r .
\end{gathered}
$$

Integration by parts formulae are important tools in infinite-dimensional analysis; see, e.g., 4]. However, the measures that are usually considered in the literature are supported by suitable topological vector spaces and give strictly positive measure to every open set. The supports of the measures that are considered in (1.2)(1.4) are instead infinite-dimensional closed convex non-affine sets. From this point of view, formulae (1.2)-(1.4) generalize the integration by parts formulae (1)-(2) of 7], which are, in fact, particular cases of (1.4) with $z=z^{\prime}$.

Consider, for instance, (1.2). The law of the Brownian Meander $M$ is naturally supported by the set of continuous nonnegative paths $x$ on $[0,1]$ that satisfy $x(0)=0$ and $x(\tau)>0$ for all $\tau \in(0,1]$. In the second term of the right-hand side of (1.2), the law of $V\left(r, b_{0}^{0}, M\right), r \in(0,1]$, is supported by the set of continuous paths $x$ on $[0,1]$ that satisfy $x(0)=x(r)=0$ and $x(\tau)>0$ for all $\tau \in(0, r) \cup(r, 1]$. Therefore, the last term in the right-hand side of (1.2) is singular with respect to the law of $M$ and can be interpreted as a boundary term. Notice also that the function $r \mapsto\left[r^{3}(1-r)\right]^{-1 / 2}$ is not integrable in any neighbourhood of 0 ; the constraints $M(0)=0$ and $M \geq 0$ produce a singularity for $r$ close to 0 of the boundary measure, which turns out to have infinite total mass.

Analogous considerations hold for (1.3) and (1.4). The supports of the laws of $V\left(r, b_{0}^{\alpha / \sqrt{r}}, M\right)$ and respectively $z+V\left(r, b_{0}^{z / \sqrt{r}}, \hat{b}_{0}^{z^{\prime} / \sqrt{1-r}}\right)$ are equal to the supports of the reference measures, i.e., the law of $B$ on $K_{\alpha}$ and respectively the law of $b_{z}^{z^{\prime}}$, with the further condition that $\{x(r)=0\}$. If $z=0$ or $z^{\prime}=0$ in (1.4), then the function of $r$ that appears in the boundary term is not integrable near 0 or respectively 1 , and the boundary measure has again infinite total mass. On the other hand, for $z, z^{\prime}>0$ in (1.4) and for all $\alpha>0$ in (1.3), the singularities disappear and the boundary measures have finite total mass. 


\section{Proof of Theorem 1.1}

We recall the following facts:

Theorem 2.1 (Denisov 11). Let $(U, M, \hat{M})$ be an independent triple such that $U: \Omega \rightarrow[0,1]$ has the arcsine law and $M$ and $\hat{M}$ are two standard Brownian Meanders. Then $V(U, M, \hat{M}) \stackrel{d}{=} B$, where $V$ is defined by (1.1).

Theorem 2.2 (Imhof [3]). The Brownian Meander $M$ is equal in law to $b_{0}^{\rho}$, where $\rho$ is independent of $\left(b_{0}^{z}\right)_{z \geq 0}$ and has the Rayleigh density

$$
\mathbb{P}(\rho \in d x)=x e^{-\frac{1}{2} x^{2}} d x, \quad x>0 .
$$

Theorem 2.3 (Durrett, Iglehart and Miller [2]). The law of $B$ conditioned to $K_{\alpha}$, $\alpha>0$, converges weakly in $E$ to the law of $M$ as $\alpha \downarrow 0$.

Without loss of generality, we can assume that

$$
h \geq 0, \quad \varphi \geq 0
$$

In particular, $K_{\alpha} \subseteq K_{\alpha}-t h$ for all $t \geq 0$. Recall that $\partial_{h} \varphi(x)=\lim _{t \downarrow 0}(\varphi(x)-$ $\varphi(x-t h)) / t$. By the Cameron-Martin theorem,

$$
\begin{aligned}
& \frac{1}{t} \mathbb{E}\left[1_{K_{\alpha}}(B)\right.(\varphi(B)-\varphi(B-t h))]=-\frac{1}{t} \mathbb{E}\left[1_{\left(K_{\alpha}-t h\right) \backslash K_{\alpha}}(B) \varphi(B)\right] \\
&+\frac{1}{t} \mathbb{E}\left[1_{K_{\alpha}-t h}(B) \varphi(B)\left(1-\exp \left(-\frac{1}{2}\left\|t h^{\prime}\right\|^{2}+t\left\langle B, h^{\prime \prime}\right\rangle\right)\right)\right]
\end{aligned}
$$

By Theorem 2.1, we have

$$
\begin{array}{r}
\mathbb{E}\left[1_{\left(K_{\alpha}-t h\right) \backslash K_{\alpha}}(B) \varphi(B)\right]=\mathbb{E}\left[1_{\left(K_{\alpha}-t h\right) \backslash K_{\alpha}}(V(U, M, \hat{M})) \varphi(V(U, M, \hat{M}))\right] \\
=\int_{0}^{1} d r \frac{1}{\pi \sqrt{r(1-r)}} \mathbb{E}\left[1_{\left(K_{\alpha}-t h\right) \backslash K_{\alpha}}(V(r, M, \hat{M})) \varphi(V(r, M, \hat{M}))\right] .
\end{array}
$$

Let $n \in \mathbb{N}, c_{n} \geq c_{n-1} \geq \cdots \geq c_{1} \geq c_{0}:=0,\left\{I_{1}, \ldots, I_{n}\right\}$ a Borel partition of $[0,1]$ and $I_{0}:=\emptyset$, and set

$$
h_{i}:=\sum_{j=1}^{n}\left(c_{j} \wedge c_{i}\right) 1_{I_{j}}, \quad i=0, \ldots, n .
$$

The key point is the following: for $i=1, \ldots, n$, since $h_{i} \geq h_{i-1}$, and $h_{i}=h_{i-1}$ on $\bigcup_{j=0}^{i-1} I_{j}$, then for all $r \in(0,1)$,

$$
\begin{aligned}
& V(r, M, \hat{M}) \in\left(K_{\alpha}-t h_{i}\right) \backslash\left(K_{\alpha}-t h_{i-1}\right) \Longleftrightarrow \\
& V(r, M, \hat{M}) \in K_{\alpha}-t h_{i}, r \in \bigcup_{j=i}^{n} I_{j} \text { and } \sqrt{r} M(1) \in\left[\alpha+t c_{i-1}, \alpha+t c_{i}\right) .
\end{aligned}
$$


Indeed, $V(r, M, \hat{M})$ attains its minimum $-\sqrt{r} M(1)$ only at time $r$. Then we obtain for all $t \geq 0$ and $i=1, \ldots, n$,

$$
\begin{aligned}
\mathbb{E} & {\left[1_{\left.\left(K_{\alpha}-t h_{i}\right) \backslash K_{\alpha}(B) \varphi(B)\right]}\right.} \\
= & \int_{0}^{1} \frac{d r}{\pi \sqrt{r(1-r)}} \mathbb{E}\left[1_{\left(K_{\alpha}-t h_{i}\right) \backslash K_{\alpha}}(V(r, M, \hat{M})) \varphi(V(r, M, \hat{M}))\right] \\
= & \int_{0}^{1} \frac{d r}{\pi \sqrt{r(1-r)}} \mathbb{E}\left[\varphi \cdot\left[1_{\left(K_{\alpha}-t h_{i-1}\right) \backslash K_{\alpha} \cup\left(K_{\alpha}-t h_{i}\right) \backslash\left(K_{\alpha}-t h_{i-1}\right)}\right](V(r, M, \hat{M}))\right] \\
= & \int_{0}^{1} \frac{d r}{\pi \sqrt{r(1-r)}} \mathbb{E}\left[\varphi \cdot 1_{\left(K_{\alpha}-t h_{i-1}\right) \backslash K_{\alpha}}(V(r, M, \hat{M}))\right] d r \\
& +\int_{\cup_{j=i}^{n} I_{j}} \frac{d r}{\pi \sqrt{r(1-r)}} \mathbb{E}\left[\varphi \cdot 1_{\left(K_{\alpha}-t h_{i}\right)}(V(r, M, \hat{M})) 1_{\left[c_{i-1}, c_{i}\right)}\left(\frac{\sqrt{r} M(1)-\alpha}{t}\right)\right] .
\end{aligned}
$$

Proceeding by induction on $n$ we obtain by Theorem 2.2

$$
\begin{aligned}
\mathbb{E} & {\left[1_{\left(K_{\alpha}-t h_{n}\right) \backslash K_{\alpha}}(B) \varphi(B)\right] } \\
& =\sum_{i=1}^{n} \int_{\cup_{j=i}^{n} I_{j}} \frac{d r}{\pi \sqrt{r(1-r)}} \mathbb{E}\left[\varphi \cdot 1_{\left(K_{\alpha}-t h_{i}\right)}(V(r, M, \hat{M})) 1_{\left[c_{i-1}, c_{i}\right)}\left(\frac{\sqrt{r} M(1)-\alpha}{t}\right)\right] \\
& =\sum_{i=1}^{n} \sum_{j=i}^{n} \int_{I_{j}} \frac{d r}{\pi \sqrt{r(1-r)}} \int_{\frac{\alpha+t c_{i-1}}{\sqrt{r}}}^{\frac{\alpha+t c_{i}}{\sqrt{r}}} y e^{-\frac{y^{2}}{2}} \mathbb{E}\left[\varphi \cdot 1_{\left(K_{\alpha}-t h_{i}\right)}\left(V\left(r, b_{0}^{y}, \hat{M}\right)\right)\right] d y .
\end{aligned}
$$

Then we obtain

$$
\begin{aligned}
\lim _{t \downarrow 0} \frac{1}{t} \mathbb{E} & {\left[1_{\left(K_{\alpha}-t h_{n}\right) \backslash K_{\alpha}}(B) \varphi(B)\right] } \\
& =\sum_{i=1}^{n} \sum_{j=i}^{n}\left(c_{i}-c_{i-1}\right) \int_{I_{j}} d r \frac{\alpha e^{-\frac{\alpha^{2}}{2 r}}}{\pi \sqrt{r^{3}(1-r)}} \mathbb{E}\left[\varphi \cdot 1_{\left(K_{\alpha}-t h_{i}\right)}\left(V\left(r, b_{0}^{y}, \hat{M}\right)\right)\right] \\
& =\sum_{j=1}^{n} \sum_{i=1}^{j}\left(c_{i}-c_{i-1}\right) \int_{I_{j}} d r \frac{\alpha e^{-\frac{\alpha^{2}}{2 r}}}{\pi \sqrt{r^{3}(1-r)}} \mathbb{E}\left[\varphi \cdot 1_{\left(K_{\alpha}\right)}\left(V\left(r, b_{0}^{\alpha / \sqrt{r}}, \hat{M}\right)\right)\right] \\
& =\int_{0}^{1} d r\left(\sum_{j=1}^{n} c_{j} 1_{I_{j}}(r)\right) \frac{\alpha e^{-\frac{\alpha^{2}}{2 r}}}{\pi \sqrt{r^{3}(1-r)}} \mathbb{E}\left[\varphi\left(V\left(r, b_{0}^{\alpha / \sqrt{r}}, \hat{M}\right)\right)\right] \\
& =\int_{0}^{1} \frac{d r}{\pi \sqrt{r^{3}(1-r)}} \alpha e^{-\frac{\alpha^{2}}{2 r}} h_{n}(r) \mathbb{E}\left[\varphi \cdot 1_{\left(K_{\alpha}-t h_{i}\right)}\left(V\left(r, b_{0}^{y / \sqrt{r}}, \hat{M}\right)\right)\right] .
\end{aligned}
$$

Now set $I_{i}:=h^{-1}([(i-1) / n, i / n)), i \in \mathbb{N}$,

$$
f_{n}:=\sum_{i=1}^{\infty} \frac{i-1}{n} 1_{I_{i}}, \quad g_{n}:=\sum_{i=1}^{\infty} \frac{i}{n} 1_{I_{i}}
$$

where both sums are finite, since $h$ is bounded. Then $f_{n} \leq h \leq g_{n}, f_{n}$ and $g_{n}$ converge uniformly on $[0,1]$ to $h$ as $n \rightarrow \infty$, and $K_{\alpha}-t f_{n} \subseteq K_{\alpha}-t h \subseteq K_{\alpha}-t g_{n}$, 
$t \geq 0$. Therefore, since $\varphi \geq 0$, we have

$$
\begin{aligned}
& \int_{0}^{1} d r f_{n}(r) \frac{\alpha \exp \left(-\frac{\alpha^{2}}{2 r}\right)}{\pi \sqrt{r^{3}(1-r)}} \mathbb{E}\left[\varphi\left(V\left(r, b_{0}^{\alpha / \sqrt{r}}, M\right)\right)\right] \\
& \quad \leq \liminf _{t \downarrow 0} \frac{1}{t} \mathbb{E}\left[1_{\left(K_{\alpha}-t h\right) \backslash K_{\alpha}}(B) \varphi(B)\right] \\
& \quad \leq \limsup _{t \downarrow 0} \frac{1}{t} \mathbb{E}\left[1_{\left(K_{\alpha}-t h\right) \backslash K_{\alpha}}(B) \varphi(B)\right] \\
& \quad \leq \int_{0}^{1} d r g_{n}(r) \frac{\alpha \exp \left(-\frac{\alpha^{2}}{2 r}\right)}{\pi \sqrt{r^{3}(1-r)}} \mathbb{E}\left[\varphi\left(V\left(r, b_{0}^{\alpha / \sqrt{r}}, M\right)\right)\right]
\end{aligned}
$$

and by (2.1),

$$
\begin{aligned}
\mathbb{E} & {\left[\partial_{h} \varphi(B) 1_{K_{\alpha}}(B)\right] } \\
& =\lim _{t \downarrow 0} \frac{1}{t} \mathbb{E}\left[1_{K_{\alpha}}(B)(\varphi(B)-\varphi(B-t h))\right] \\
& =-\mathbb{E}\left[\varphi(B)\left\langle B, h^{\prime \prime}\right\rangle 1_{K_{\alpha}}(B)\right]-\int_{0}^{1} d r \frac{h(r) \alpha e^{-\frac{\alpha^{2}}{2 r}}}{\pi \sqrt{r^{3}(1-r)}} \mathbb{E}\left[\varphi\left(V\left(r, b_{0}^{\alpha / \sqrt{r}}, M\right)\right)\right]
\end{aligned}
$$

so that (1.3) is proved. In order to prove (1.2), we recall that

$$
\begin{aligned}
\mathbb{P}\left(B \in K_{\alpha}\right) & =\mathbb{P}\left(\inf _{[0,1]} B \geq-\alpha\right)=\mathbb{P}\left(\left|B_{1}\right| \leq \alpha\right) \\
& =\sqrt{\frac{2}{\pi}} \int_{0}^{\alpha} e^{-\frac{y^{2}}{2}} d y \sim \sqrt{\frac{2}{\pi}} \alpha
\end{aligned}
$$

as $\alpha \rightarrow 0$. We divide (1.3) by $\mathbb{P}\left(B \in K_{\alpha}\right)$. Since $h$ has compact support in $(0,1)$ and the laws of $e_{0, \alpha}^{r}$ are weakly continuous in $\alpha \geq 0$, we can let $\alpha \downarrow 0$ in the last term of (1.3). Then we apply Theorem 2.3 to the first and second term in (1.3) and the proof of (1.2) is complete.

Now we prove (1.4). We choose $h=k \in C_{c}^{2}(0,1)$. Since $k$ has compact support in $(0,1)$, we have for all $z \geq 0$,

$$
\left\langle B, k^{\prime \prime}\right\rangle=\left\langle B+z, k^{\prime \prime}\right\rangle-z\left(k^{\prime}(1)-k^{\prime}(0)\right)=\left\langle B+z, k^{\prime \prime}\right\rangle .
$$

Therefore, we can write (1.3) in the following way:

$$
\begin{aligned}
\mathbb{E}\left[\partial_{k} \varphi(B+z) 1_{K_{0}}(B+z)\right]=-\mathbb{E}\left[\varphi(B+z)\left\langle B+z, k^{\prime \prime}\right\rangle 1_{K_{0}}(B+z)\right] \\
-\int_{0}^{1} d r k(r) \frac{z \exp \left(-\frac{z^{2}}{2 r}\right)}{\pi \sqrt{r^{3}(1-r)}} \mathbb{E}\left[\varphi\left(z+V\left(r, b_{0}^{z / \sqrt{r}}, M\right)\right)\right] .
\end{aligned}
$$

For all $\epsilon>0$, let $\gamma^{\epsilon} \in C^{1}(\mathbb{R})$ such that

$$
0 \leq \gamma^{\epsilon} \leq 1, \gamma^{\epsilon} \equiv 1 \text { over }[-\epsilon, \epsilon], \gamma^{\epsilon} \equiv 0 \text { over } \mathbb{R} \backslash\left[-\epsilon-\epsilon^{2}, \epsilon+\epsilon^{2}\right] .
$$

We set for $z, z^{\prime} \geq 0$ and $\epsilon>0$,

$$
\psi^{\epsilon}: E \mapsto \mathbb{R}, \quad \psi^{\epsilon}(x):=\frac{\gamma^{\epsilon}\left(x(1)-z^{\prime}\right)}{\mathbb{E}\left[\gamma^{\epsilon}\left(B(1)+z-z^{\prime}\right) \cdot 1_{K_{0}}(B+z)\right]}
$$


Then we have

$$
\partial_{k}\left[\varphi \cdot \psi^{\epsilon}\right](x)=\left[\partial_{k} \varphi\right](x) \cdot \psi^{\epsilon}(x)+\frac{\left[\gamma^{\epsilon}\right]^{\prime}\left(x(1)-z^{\prime}\right) \cdot k(1)}{\mathbb{E}\left[\gamma^{\epsilon}\left(B(1)+z-z^{\prime}\right) \cdot 1_{K_{0}}(B+z)\right]} .
$$

Since $k$ has compact support in $(0,1)$, we have $k(1)=0$, and therefore,

$$
\begin{aligned}
& \mathbb{E}\left[\left[\left(\partial_{k} \varphi\right) \cdot \psi^{\epsilon}\right](B+z) 1_{K_{0}}(B+z)\right] \\
& =-\mathbb{E}\left[\varphi(B+z)\left\langle B+z, k^{\prime \prime}\right\rangle 1_{K_{0}}(B+z)\right] \\
& \quad-\int_{0}^{1} d r k(r) \frac{z \exp \left(-\frac{z^{2}}{2 r}\right)}{\pi \sqrt{r^{3}(1-r)}} \mathbb{E}\left[\left[\cdot \cdot \psi^{\epsilon}\right]\left(z+V\left(r, b_{0}^{z / \sqrt{r}}, M\right)\right)\right] .
\end{aligned}
$$

Notice now that

$$
\begin{aligned}
& \psi^{\epsilon}\left(z+V\left(r, b_{0}^{z / \sqrt{r}}, M\right)\right) \\
& \quad=\frac{\gamma^{\epsilon}\left(\sqrt{1-r} M(1)-z^{\prime}\right)}{\mathbb{E}\left[\gamma^{\epsilon}\left(\sqrt{1-r} M(1)-z^{\prime}\right)\right]} \cdot \frac{\mathbb{E}\left[\gamma^{\epsilon}\left(\sqrt{1-r} M(1)-z^{\prime}\right)\right]}{\mathbb{E}\left[\gamma^{\epsilon}\left(B(1)+z-z^{\prime}\right) \cdot 1_{K_{0}}(B+z)\right]} .
\end{aligned}
$$

By the Reflection Principle (see, e.g., III.3.14-(4) in $[6]$ ), for all $f: \mathbb{R} \mapsto \mathbb{R}$ bounded and Borel we have

$$
\mathbb{E}\left[f(B(1)+z) \cdot 1_{K_{0}}(B+z)\right]=\frac{1}{\sqrt{2 \pi}} \int_{0}^{+\infty} f(y)\left(e^{-(z-y)^{2} / 2}-e^{-(z+y)^{2} / 2}\right) d y .
$$

Moreover, by Theorem 2.2 $M(1)$ has the Rayleigh density $x e^{-\frac{1}{2} x^{2}} d x$ on $\{x>0\}$. Then

$$
\begin{aligned}
& \lim _{\epsilon \downarrow 0} \frac{\mathbb{E}\left[\gamma^{\epsilon}\left(\sqrt{1-r} M(1)-z^{\prime}\right)\right]}{\mathbb{E}\left[\gamma^{\epsilon}\left(B(1)+z-z^{\prime}\right) \cdot 1_{K_{0}}(B+z)\right]} \\
& =\lim _{\epsilon \downarrow 0} \frac{\sqrt{2 \pi} \int_{\left(z^{\prime}-\epsilon\right) / \sqrt{1-r}}^{\left(z^{\prime}+\epsilon\right)} y e^{-y^{2} / 2} d y}{\int_{z^{\prime}-\epsilon}^{z^{\prime}+\epsilon}\left(e^{-(z-y)^{2} / 2}-e^{-(z+y)^{2} / 2}\right) d y}=\frac{\sqrt{2 \pi} z^{\prime} \exp \left(-\frac{\left(z^{\prime}\right)^{2}}{2(1-r)}+\frac{\left(z-z^{\prime}\right)^{2}}{2}\right)}{(1-r)\left(1-\exp \left(-2 z z^{\prime}\right)\right)} .
\end{aligned}
$$

Therefore, by Theorem 2.2. letting $\epsilon \downarrow 0$ in (2.3) for all $z, z^{\prime}>0$, we obtain

$$
\begin{aligned}
\mathbb{E}\left[\partial_{k} \varphi\left(b_{z}^{z^{\prime}}\right)\right]= & -\mathbb{E}\left[\varphi\left(b_{z}^{z^{\prime}}\right)\left\langle b_{z}^{z^{\prime}}, k^{\prime \prime}\right\rangle\right] \\
- & \int_{0}^{1} d r k(r) \frac{\exp \left(-\frac{z^{2}}{2} \frac{r}{1-r}-\frac{\left(z^{\prime}\right)^{2}}{2} \frac{1-r}{r}-2 z z^{\prime}\right)}{\sqrt{2 \pi r^{3}(1-r)^{3}}} \frac{2 z z^{\prime}}{1-\exp \left(-2 z z^{\prime}\right)} \\
\cdot & \mathbb{E}\left[\varphi\left(z+V\left(r, b_{0}^{z / \sqrt{r}}, \hat{b}_{0}^{z^{\prime} / \sqrt{1-r}}\right)\right)\right]
\end{aligned}
$$

and (1.4) is proved.

\section{SPDES WITH REFLECTION}

Arguing as in [7, the formulae (1.2)-(1.4) find applications to SPDEs with reflection (see [5]). Let $\{W(t, \theta): t \geq 0, \theta \in[0,1]\}$ be a Brownian Sheet. 
Theorem 3.1. For all $\alpha \geq 0$ let $E_{\alpha}:=\{x:[0,1] \mapsto[-\alpha, \infty)$ continuous, $x(0)=0\}$, and for all $x \in E_{\alpha}$ let $\left(v_{\alpha}, \zeta_{\alpha}\right)$ be the solution of the following SPDE with reflection:

$$
\left\{\begin{array}{l}
\frac{\partial v_{\alpha}}{\partial t}=\frac{1}{2} \frac{\partial^{2} v_{\alpha}}{\partial \theta^{2}}+\frac{\partial^{2} W}{\partial t \partial \theta}+\zeta_{\alpha}, \\
v_{\alpha}(0, \theta)=x(\theta), v_{\alpha}(t, 0)=\frac{\partial v_{\alpha}}{\partial \theta}(t, 1)=0, \\
v_{\alpha}+\alpha \geq 0, d \zeta_{\alpha} \geq 0, \int\left(v_{\alpha}+\alpha\right) d \zeta_{\alpha}=0,
\end{array}\right.
$$

where $v_{\alpha}:=[0, \infty) \times[0,1] \mapsto \mathbb{R}$ is continuous and $\zeta_{\alpha}$ is a locally finite positive measure on $[0, \infty) \times(0,1)$. We write $v_{\alpha}=v_{\alpha}(t, \theta, x), t \geq 0, \theta \in[0,1], x \in E_{\alpha}$. Also, let $m_{\alpha}$ be the law of $B$ conditioned to $E_{\alpha}$ for $\alpha>0$ and the law of $M$ for $\alpha=0$. Then for all $\alpha \geq 0$ :

1. The process $\left(v_{\alpha}(t, \cdot, x)\right)_{t \geq 0, x \in E_{\alpha}}$ is symmetric with respect to its unique invariant probability measure $m_{\alpha}$.

2. The process $\left(v_{\alpha}(t, \cdot, x)\right)_{t \geq 0, x \in E_{\alpha}}$ is the Markov process properly associated with the symmetric Dirichlet Form $\left(\Gamma^{\alpha}, D\left(\Gamma^{\alpha}\right)\right)$ in $L^{2}\left(m_{\alpha}\right)$, and the closure of the bilinear form

$$
C_{b}^{1}\left(L^{2}(0,1)\right) \ni \varphi, \psi \mapsto \frac{1}{2} \int_{E_{\alpha}}\langle\nabla \varphi, \nabla \psi\rangle d m_{\alpha} .
$$

3. For all Borel sets $I \subset \subset(0,1)$, the process $t \mapsto \zeta_{\alpha}([0, t] \times I)$ is an Additive Functional of $v_{\alpha}$, with Revuz-measure

$$
\begin{gathered}
\alpha>0: \quad \int_{E_{\alpha}} \mathbb{E}\left[\int_{0}^{1} \varphi\left(v_{\alpha}(t, \cdot, x)\right) \zeta_{\alpha}(d t, I)\right] m_{\alpha}(d x) \\
=\frac{1}{2} \frac{\alpha}{\int_{0}^{\alpha} e^{-\frac{y^{2}}{2}} d y} \int_{I} \frac{\exp \left(-\frac{\alpha^{2}}{2 r}\right)}{\sqrt{2 \pi r^{3}(1-r)}} \mathbb{E}\left[\varphi\left(V\left(r, b_{0}^{\alpha / \sqrt{r}}, M\right)\right)\right] d r, \\
\alpha=0: \quad \int_{K_{0}} \mathbb{E}\left[\int_{0}^{1} \varphi\left(v_{0}(t, \cdot, x)\right) \zeta_{0}(d t, I)\right] m_{0}(d x) \\
=\frac{1}{2} \int \frac{1}{\sqrt{2 \pi r^{3}(1-r)}} \mathbb{E}\left[\varphi\left(V\left(r, b_{0}^{0}, M\right)\right)\right] d r,
\end{gathered}
$$

for $\varphi: L^{2}(0,1) \mapsto \mathbb{R}$ Borel and bounded.

4. For all $x \in E_{\alpha}$, there exist a random Borel set $S_{\alpha} \subset \mathbb{R}_{+}$and a map $r_{\alpha}: S_{\alpha} \mapsto(0,1)$, such that a.s.

$$
\begin{array}{ll} 
& \zeta_{\alpha}\left(\left[\mathbb{R}_{+} \times(0,1)\right] \backslash\left\{\left(s, r_{\alpha}(s)\right): s \in S_{\alpha}\right\}\right)=0 \\
\forall s \in S_{\alpha}: & v_{\alpha}\left(s, r_{\alpha}(s)\right)=0, \quad v_{\alpha}(s, \theta)>0 \quad \forall \theta \in(0,1) \backslash\left\{r_{\alpha}(s)\right\} .
\end{array}
$$

5. Let $\delta_{r}$ denote the Dirac mass at $r \in(0,1)$. For all $x \in E_{\alpha}$, we have a.s. on $[0, \infty) \times(0,1)$,

$$
\zeta_{\alpha}(d s, d \theta)=\delta_{r_{\alpha}(s)}(d \theta) \zeta_{\alpha}(d s,(0,1)) .
$$

Theorem 3.2. For all $z, z^{\prime} \geq 0$ let $D_{z}^{z^{\prime}}:=\{x:[0,1] \mapsto[0, \infty)$ continuous, $x(0)=$ $\left.z, x(1)=z^{\prime}\right\}$, and for all $x \in D_{z}^{z^{\prime}}$ let $\left(w_{z}^{z^{\prime}}, \gamma_{z}^{z^{\prime}}\right)$ be the solution of the following 
SPDE with reflection:

$$
\left\{\begin{array}{l}
\frac{\partial w_{z}^{z^{\prime}}}{\partial t}=\frac{1}{2} \frac{\partial^{2} w_{z}^{z^{\prime}}}{\partial \theta^{2}}+\frac{\partial^{2} W}{\partial t \partial \theta}+\gamma_{z}^{z^{\prime}} \\
w_{z}^{z^{\prime}}(0, \theta)=x(\theta), w_{z}^{z^{\prime}}(t, 0)=z, w_{z}^{z^{\prime}}(t, 1)=z^{\prime} \\
w_{z}^{z^{\prime}} \geq 0, d \gamma_{z}^{z^{\prime}} \geq 0, \int w_{z}^{z^{\prime}} d \gamma_{z}^{z^{\prime}}=0
\end{array}\right.
$$

where $w_{z}^{z^{\prime}}:=[0, \infty) \times[0,1] \mapsto \mathbb{R}$ is continuous and $\gamma_{z}^{z^{\prime}}$ is a locally finite positive measure on $[0, \infty) \times(0,1)$. We write $w_{z}^{z^{\prime}}=w_{z}^{z^{\prime}}(t, \theta, x), t \geq 0, \theta \in[0,1], x \in D_{z}^{z^{\prime}}$. Also, let $n_{z}^{z^{\prime}}$ be the law of $b_{z}^{z^{\prime}}$. Then for all $z, z^{\prime} \geq 0$ :

1. The process $\left(w_{z}^{z^{\prime}}(t, \cdot, x)\right)_{t \geq 0, x \in D_{z}^{z^{\prime}}}$ is symmetric with respect to its unique invariant probability measure $n_{z}^{z^{\prime}}$.

2. The process $\left(w_{z}^{z^{\prime}}(t, \cdot, x)\right)_{t \geq 0, x \in D_{z}^{z^{\prime}}}$ is the Markov process properly associated with the symmetric Dirichlet Form $\left(\Gamma^{z, z^{\prime}}, D\left(\Gamma^{z, z^{\prime}}\right)\right)$ in $L^{2}\left(n_{z}^{z^{\prime}}\right)$, and the closure of the bilinear form:

$$
C_{b}^{1}\left(L^{2}(0,1)\right) \ni \varphi, \psi \mapsto \frac{1}{2} \int_{D_{z}^{z^{\prime}}}\langle\nabla \varphi, \nabla \psi\rangle d n_{z}^{z^{\prime}} .
$$

3. For all Borel sets $I \subset \subset(0,1)$, the process $t \mapsto \gamma_{z}^{z^{\prime}}([0, t] \times I)$ is an Additive Functional of $w_{z}^{z^{\prime}}$, with Revuz-measure

$$
\begin{aligned}
& \int_{D_{z}^{z^{\prime}}} \mathbb{E}\left[\int_{0}^{1} \varphi\left(w_{z}^{z^{\prime}}(t, \cdot, x)\right) \gamma_{z}^{z^{\prime}}(d t, I)\right] n_{z}^{z^{\prime}}(d x) \\
&=\frac{1}{2} \frac{2 z z^{\prime}}{1-\exp \left(-2 z z^{\prime}\right)} \int_{I} \frac{\exp \left(-\frac{z^{2}}{2} \frac{r}{1-r}-\frac{\left(z^{\prime}\right)^{2}}{2} \frac{1-r}{r}-2 z z^{\prime}\right)}{\sqrt{2 \pi r^{3}(1-r)^{3}}} . \\
& \cdot \mathbb{E}\left[\varphi\left(z+V\left(r, b_{0}^{z / \sqrt{r}}, \hat{b}_{0}^{z^{\prime} / \sqrt{1-r}}\right)\right)\right] d r,
\end{aligned}
$$

for $\varphi: L^{2}(0,1) \mapsto \mathbb{R}$ Borel and bounded.

4. For all $x \in D_{z}^{z^{\prime}}$, there exist a random Borel set $S_{z}^{z^{\prime}} \subset \mathbb{R}_{+}$and a map $r_{z}^{z^{\prime}}: S_{z}^{z^{\prime}} \mapsto(0,1)$, such that a.s.

$$
\begin{gathered}
\gamma_{z}^{z^{\prime}}\left(\left[\mathbb{R}_{+} \times(0,1)\right] \backslash\left\{\left(s, r_{z}^{z^{\prime}}(s)\right): s \in S_{z}^{z^{\prime}}\right\}\right)=0 \\
\forall s \in S_{z}^{z^{\prime}}: \quad w_{z}^{z^{\prime}}\left(s, r_{z}^{z^{\prime}}(s)\right)=0, \quad w_{z}^{z^{\prime}}(s, \theta)>0 \quad \forall \theta \in(0,1) \backslash\left\{r_{z}^{z^{\prime}}(s)\right\} .
\end{gathered}
$$

5. Let $\delta_{r}$ denote the Dirac mass at $r \in(0,1)$. For all $x \in D_{z}^{z^{\prime}}$, we have a.s. on $[0, \infty) \times(0,1)$ :

$$
\gamma_{z}^{z^{\prime}}(d s, d \theta)=\delta_{r_{z}^{\prime}(s)}(d \theta) \gamma_{z}^{z^{\prime}}(d s,(0,1))
$$

\section{REFERENCES}

[1] I. V. Denisov, (1983), A random walk and a Wiener process near a maximum, Theor. Probab. Appl., 28, 821-824. MR 85f:60117

[2] R. T. Durrett, D. L. Iglehart, and D. R. Miller, (1977), Weak convergence to Brownian meander and Brownian excursion, Ann. Probability, 5, no. 1, 117-129. MR 55:9300 
[3] J. P. Imhof, (1984), Density factorizations for Brownian motion, meander and the threedimensional Bessel process, and applications, J. Appl. Probab., 21, no. 3, 500-510. MR 85j:60152

[4] P. Malliavin, (1997), Stochastic Analysis, Grundlehren der mathematischen Wissenschaften, Band 313, Springer-Verlag, Berlin, Heidelberg, New York. MR 99b:60073

[5] D. Nualart and E. Pardoux, (1992), White noise driven quasilinear SPDEs with reflection, Probab. Theory and Related Fields, 93, 77-89. MR 93h:60093

[6] D. Revuz and M. Yor, (1991), Continuous Martingales and Brownian Motion, Grundlehren der mathematischen Wissenschaften, Band 293, Springer-Verlag, Berlin. MR 92d:60053

[7] L. Zambotti, (2002), Integration by parts formulae on convex sets of paths and applications to SPDEs with reflection, Probab. Theory and Related Fields, 123, no. 4, 579-600. MR 2003e: 60120

Dipartimento di Matematica, Università di Trento, Via Sommarive 14, 38050 Povo (TRENTO), ITALY

E-mail address: bonaccor@science.unitn.it

Scuola Normale Superiore, Piazza dei Cavalieri 7, 56126 Pisa, Italy

E-mail address: zambotti@sns.it 